\title{
ArcheoSciences
}

Revue d'archéométrie

\section{Assèchement et dégradation des sols durant le Subatlantique : vers un niveau repère antique dans le Nord-Est de la France (Lorraine, Alsace)?}

Soil dessiccation and degradation during the Subatlantic: towards an antic soil marker (reference layer) in North-Eastern France (Lorraine, Alsace)?

\section{Anne Gebhardt, Anaïs Champougny et Patrice Wuscher}

\section{OpenEdition \\ Journals}

\author{
Édition électronique \\ URL : https://journals.openedition.org/archeosciences/5954 \\ DOI : 10.4000/archeosciences.5954 \\ ISBN : 978-2-7535-7732-9 \\ ISSN : 2104-3728 \\ Éditeur \\ Presses universitaires de Rennes
}

\section{Édition imprimée}

Date de publication : 31 décembre 2018

Pagination : 77-94

ISBN : 978-2-7535-7730-5

ISSN : 1960-1360

Référence électronique

Anne Gebhardt, Anaïs Champougny et Patrice Wuscher, « Assèchement et dégradation des sols durant le Subatlantique : vers un niveau repère antique dans le Nord-Est de la France (Lorraine, Alsace) ? », ArcheoSciences [En ligne], 42-2 | 2018, mis en ligne le 13 février 2020, consulté le 27 janvier 2022. URL : http://journals.openedition.org/archeosciences/5954 ; DOI : https://doi.org/10.4000/ archeosciences.5954 


\title{
Assèchement et dégradation des sols durant le Subatlantique : vers un niveau repère antique dans le Nord-Est de la France (Lorraine, Alsace)?
}

\author{
Soil Dessiccation and Degradation during the Subatlantic: Towards an Antic Soil \\ Marker (Reference Layer) in North-Eastern France (Lorraine, Alsace)?
}

\author{
Anne Gebhardta ${ }^{\mathrm{a}}$, Anaïs Champougny ${ }^{\mathrm{a}}$ et Patrice Wuscher ${ }^{\mathrm{b}}$
}

\begin{abstract}
Résumé : Dans le Grand Est de la France, les opérations d'archéologie préventive mettent fréquemment au jour un petit réseau polygonal de faible amplitude. Associé à des digitations blanchâtres il a souvent été interprété comme un réseau de petites fentes de gel périglaciaires. Mais à l'occasion de quatre fouilles archéologiques, nous avons noté que ce réseau s'est développé dans un sédiment mis en place entre la fin de l'Âge du Fer et le haut Moyen Âge. Ces observations remettent en question l'origine périglaciaire systématique de ce type de réseau. Après avoir passé en revue les différentes modalités de formation de telles digitations polygonales, l'hypothèse d'un développement de fentes de sécheresse lors de stress hydrique holocène au sein de sédiments argilo-limoneux saturés en eau est défendue. Enfin une discussion sur l'origine de cette dessiccation montre qu'elle semble liée à la conjonction de processus anthropiques et d'une oscillation climatique.
\end{abstract}

\begin{abstract}
In north-eastern France, minor-range polygonal networks with finger-like structures or digitations are frequently observed during archaeological fieldworks and were commonly interpreted as small periglacial networks of frost cracks. From the study of four archeological sites, this type of minor network is developped in a sediment of an age constrained between the end of the Iron Age and Early Middle Age, and the periglacial origin systematically given is questionned. The origin of such polygonal digitations in Late Holocene soils is related to a network of desiccation cracks developed under hydric stress and drying-out of water-saturated clay-loamy sediments. The origins of this well marked regional desiccation of soils seem both anthropogenic and climatic.
\end{abstract}

Mots clés : Antiquité, dessiccation des sols, Grand Est, France, repère pédo-sédimentaire.

Keywords: Antic period, Grand-Est Region, north-eastern France, France, soil desiccation, soil marker.

\section{INTRODUCTION}

Les fentes de gel et le réseau polygonal qui leur est associé ont depuis longtemps fait l'objet d'études approfondies en Europe (Van Vliet-Lanoë, 1984, 1988, 2006; Ewertowski,
2009) et sont souvent observées par le géomorphologue à l'occasion de diagnostics archéologiques (Lenoble et al., 2012). Ainsi, lors du suivi de travaux d'archéologie préventive dans l'Est de la France, faute de calages chronologiques, de petits réseaux polygonaux de plus ou moins faible ampli-

\footnotetext{
a Université de Strasbourg, CNRS, LIVE UMR; université de Lorraine, CNRS, LIEC UMR 7360, INRAP Grand Est. (anne.gebhardt-even@inrap.fr) (anais.champougny@inrap.fr)

${ }^{b}$ Université de Strasbourg, CNRS, LIVE UMR 7362, Archéologie Alsace. (patrice.wuscher@archeologie.alsace)
} 
tude ont donc naturellement été associés à des petites fentes périglaciaires provoquées par le gel. Mais sur un site localisé en bordure du lit majeur de la Seille, des traces similaires ont été découvertes au sommet d'une épaisse séquence sédimentaire colluviale datée entre l'Âge du Bronze et la fin de l'Âge du Fer. Cette observation exclut donc l'hypothèse d'une mise en place du réseau polygonal en contexte périglaciaire antérieure à l'Holocène.

Dans cet article, nous montrerons qu'il peut s'agir d'une dégradation résultant d'un stress hydrique lié à l'assèchement de sédiments limono-argileux. À ce jour, ce type de figure pédologique est observé plutôt en bas de versant et en bordure de milieu humide. Une attention particulière portée à cet événement pédologique, sur 4 sites archéologiques, a permis un premier calage chronologique et, ainsi, de le proposer comme niveau repère pour les futurs diagnostics archéologiques. Enfin, en confrontant ces observations aux données climatiques et historiques disponibles pour la région, cet exposé sera également l'occasion d'aborder l'origine de cet épisode d'assèchement des sols qui semble bien marqué en Alsace et en Lorraine.

\section{ConteXte géomorphologiQue}

La région étudiée (fig. 1) s'articule autour du massif des Vosges et s'étend de la partie orientale du bassin de Paris, à l'ouest, au fossé rhénan à l'est. En Lorraine, la couverture mésozoïque forme un paysage caractéristique de côtes calcaires orientées nord/sud structurant le réseau hydrographique principal (Moselle, Meurthe et Sarre; Lexa-Chomard et Pautrot, 2006; Le Roux, 2007). En Alsace, la formation d'un graben nord/sud au cours de l'ère tertiaire contraint les cours du Rhin et de l'Ill, et crée, au pied du massif, un relief de collines sous-vosgiennes essentiellement calcaires (Sell et al., 1998; Vogt, 1992). Les formations superficielles (Carcaud, 1992; Vogt, 1992; Sell et al., 1998; Flageollet, 2002; Cordier et al., 2004) sont principalement issues des glaciations quaternaires (dépôts alluviaux de fond de vallée ou en terrasses et limons éoliens), mais on y rencontre également des dépôts de pente tertiaires et quaternaires. Le relief impose au climat un faible gradient, de tempéré à légèrement continental en Lorraine, à plus continental en Alsace. La pluviométrie s'échelonne entre $750 \mathrm{~mm} / \mathrm{an}$ le long de la vallée moyenne de la Moselle, $2400 \mathrm{~mm} / \mathrm{an}$ au Ballon d'Alsace et autour de $600 \mathrm{~mm} / \mathrm{an}$ dans la plaine d'Alsace. (Sell et al., 1998; Wahl, 2007).

$\mathrm{Du}$ fait de ces caractères géologiques, géomorphologiques et climatiques, la brunification et le lessivage apparaissent comme les processus pédologiques principaux lorsque les sols sont bien drainés; brunisols et luvisols (Baize et Girard, 2009) ont donc une large extension (Jamagne, 2011). Les couches tertiaires argileuses, les épaisses altérites lorraines, les sols pléistocènes évolués et anciens sur limons lœssiques, le caractère faillé de la bordure vosgienne du fossé rhénan et la hauteur de la nappe phréatique dans le remplissage alluvial pléistocène de la plaine du Rhin induisent toutefois une hydromorphie importante qui prend fréquemment le pas sur le lessivage et la brunification.

\section{Méthodologie}

L'acquisition des données s'est faite au cours de dix années de suivi géomorphologique de chantiers de fouilles et de diagnostics d'archéologie préventive menés en Alsace et en Lorraine par l'INRAP et Archéologie Alsace, en interaction avec les prescriptions des Services Régionaux de l'Archéologie. Létude géomorphologique et l'analyse pédo-sédimentaire des successions stratigraphiques de 4 sites (fig. 1) datés par du matériel archéologique et/ou des datations absolues, permet de proposer un premier calage chronologique de ce petit réseau polygonale. Un inventaire des réseaux à fentes blanchâtres repérés lors des opérations d'archéologie préventive permet d'en repérer la répartition spatiale, même si leur calage chronologique n'est pas toujours confirmé.

Un inventaire des digitations polygonales repérées à ce jour en contexte de diagnostic dans le Grand Est a été listé sous la forme d'un tableau détaillant leurs caractéristiques spécifiques : angle de connexion -T ou Y-, profondeur maximale, largeur moyenne des fentes, caractéristiques sédimentaires, position topographique, corrélation archéologique, type de sol, utilisation agricole actuelle, présence de drain, chronologie...

\section{RÉSUltats}

\section{Description des sites archéologiques de référence}

\section{Bouxières-sous-Froidemont (Meurthe-et-Moselle)}

Le site de Bouxières-sous-Froidemont (Deffressigne et al., 2012) est situé sur les pentes d'un petit relief (alt. $188 \mathrm{~m}$ ) composé de marnes liasiques (argiles du Domérien) encadrant un thalweg qui s'ouvre, une dizaine de mètres plus bas, sur un paléochenal en bordure du lit majeur de la Seille (fig. 1, site 8; fig. 2a). Une première occupation s'installe sur le bord de ce chenal dès l'Âge du Bronze. Une planche reposant sur les argiles grises gleyifiées du fond de ce dernier est datée de $2895+/-40$ BP $(1095+/-68$ cal BC, Âge du Bronze final IIb). À La Tène D2 (-27 avant notre ère/+ 14 de 


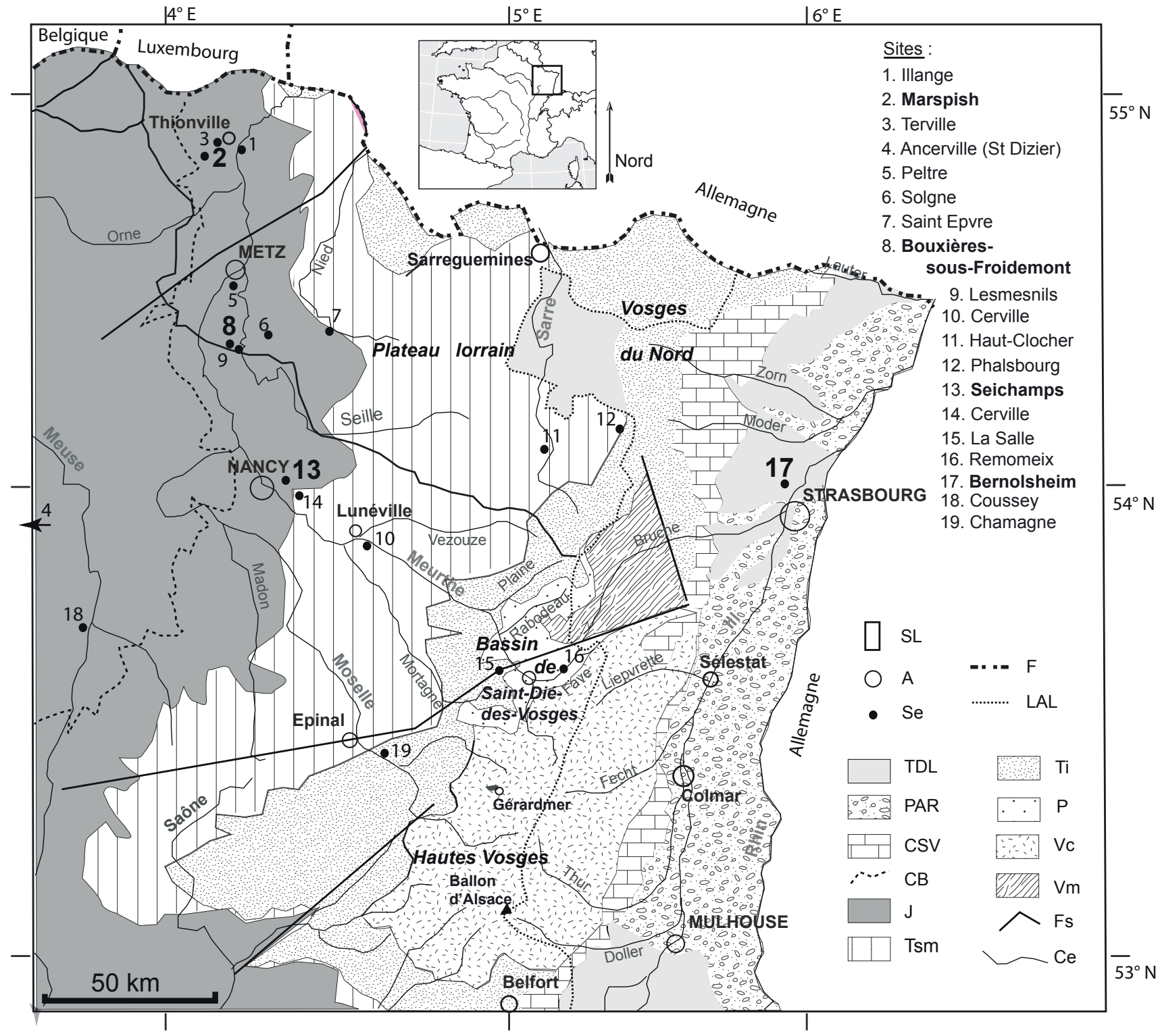

Figure 1 : localisation des sites étudiés, sur la carte géologique simplifiée de la région Grand Est, d'après Lexa-Chomard et Pautrot (2006) et Sell et al. (1998). SL : Sillon lorrain, A : Agglomérations, Se : Sites étudiés, F : Frontières, LAL : Limite Alsace/Lorraine, TDL : Terrasses et dépôts loessiques, PAR : Plaine alluviale rhénane, CSV : Collines sous vosgiennes, CB : Côte bajocienne, J : Jurassique, Tsm : Trias supérieur (Keuper) et moyen (calcaires et marnes calcaires du Muschelkalk), Ti : Trias inférieur (Grès du Buntsandstein), P : Permien (Grès) Bassin de Saint-Dié-des-Vosges, Vc : Socle des Vosges cristallines, Vm : Socle des Vosges moyennes métamorphiques, Fs : Failles structurantes principales, Ce : Cours d'eau.

Figure 1: location of the studied sites, on the geological map of the Grand Est Region (simplified from Lexa-Chomard and Pautrot (2006) et Sell et al. (1998). SL: Sillon lorrain, A: Cities, Se: Studied Sites, F. Country border, LAL: Alsace/Lorraine border, TDL: Terraces and loess deposits, PAR: Rhine Alluvial Plain, CSV: Vosgian hills, CB: Bajocian cuesta, J: Jurassic, Tsm: Upper (Keuper) and Middle (Muschelkalk) Trias, Ti: Lower Trias (Buntsandstein), P: Permian (Sandstone) Saint-Dié-des-Vosges Basin, Vc: Crystallines Vosges basement, Vm: Middle Vosges metamorphic basement, Fs: Main structural faults, Ce: river. 
notre ère), un bâtiment sur poteaux est construit sur le comblement du chenal abandonné (bât. 30). Enfin, au milieu $\mathrm{du} \mathrm{i}^{\text {er }}$ siècle de notre ère, une ferme familiale, perdurant jusqu'à la fin du $\mathrm{IV}^{\mathrm{e}}$ siècle de notre ère, est construite sur une petite proéminence à proximité (site 20). Des dépendances (bât. 25, seconde moitié du $\mathrm{I}^{\mathrm{er}}$ siècle/première moitié du II $^{\mathrm{e}}$ siècle de notre ère) ainsi qu'un fossé de drainage permettant l'écoulement des eaux vers le chenal y sont associés. Une nécropole contemporaine de cette occupation est identifiée sur un autre bombement, bien au-dessus de la cote d'inondation (S3, tumulus hallstattien et incinérations gallo-romaines).

Dans la séquence sédimentaire limono-argileuse qui recouvre le site sur environ $1 \mathrm{~m}$ de puissance à la fin de l'Âge du Fer, un petit réseau blanchâtre réticulé en plan et digité en coupe a été observé (fig. 2b, c, d). Ce réseau apparaît au sommet des niveaux antiques et ses digitations s'enfoncent sur $50 \mathrm{~cm}$ à travers les niveaux de La Tène finale puis $\mathrm{du}$ Bronze final.

\section{Marspisch (Moselle)}

Le hameau de Marspisch (Mondy et al., 2015) est implanté sur le flanc nord-ouest d'une colline culminant à 209 mètres, et borde un petit ruisseau affluent de la Fensch (fig. 1, site 2; fig. 3a). Le substrat, recouvert localement par des limons quaternaires, correspond à des marnes et des grès liasiques (Toarcien) ainsi qu'à des formations ferrugineuses (Aalénien). Un poteau de bois daté de - 56 avant notre ère par la dendrochronologie correspond au vestige le plus ancien du site. Différents types de fonds de cabane et un bas-fourneau ont été datés entre le haut Moyen Âge (bât. 13, $\mathrm{VI}^{\mathrm{e}} / \mathrm{VII}^{\mathrm{e}}$ siècle) et le $\mathrm{XII}^{\mathrm{e}}$ siècle. Le hameau se structure autour d'axes de circulation, dont un ancien thalweg (st. 1403, fig. 3c) qui se transforme par érosion en un chemin creux et finit par se combler pour disparaître complètement du paysage au XII siècle suite à l'abandon du hameau. Le réseau digité polygonal, qui épouse la structuration concentrique du sédiment liée à l'enfoncement du poteau, doit être considéré comme postérieur à ce dernier daté de La Tène finale (fig. 3b). Il est recouvert par le niveau mérovingien et recoupé par un second chemin creux (st. 1366, fig. 3b) qui se raccorde au thalweg principal (st. 1043). Ce dernier commence à se remblayer au viII ${ }^{\mathrm{e}}$ siècle pour être colmaté quatre siècles plus tard. Le réseau digité de Marspisch peut donc être calé entre La Tène finale et le viI ${ }^{\mathrm{e}}$ siècle.

\section{Bernolsheim (Bas-Rhin)}

Le site de Bernolsheim (fig. 1, site 17; fig. 4a) se situe au pied de collines loessiques, sur une terrasse alluviale gravelosableuse qui domine de plus de 5 mètres la Zorn, important cours d'eau d'origine vosgienne. Attribué à l'avant dernièreglaciation sur la base de sa position stratigraphique (Blanalt et al., 1972), un sol argileux évolué s'est développé sur cette terrasse. Vers le versant, cette terrasse est recouverte par un glacis composé de dépôts de pente, de limons loessiques et de paléosols pléistocènes. Le sol évolué, les paléosols pléistocènes et certains dépôts de pente conditionnent les écoulements de surface et de sub-surface. Les vestiges archéologiques nombreux et denses présents sur cette terrasse ont été explorés sur plus de 100 hectares (Fleicher et al., 2011; Leprovost et Fleischer, 2014; Fleischer et Leprovost, 2015). Les occupations les plus anciennes sont matérialisées par un village du Néolithique ancien (environ 5400 à -4900 ans avant notre ère), puis par un village et une nécropole du Néolithique moyen (environ 4900-4600 ans avant notre ère). Au Bronze moyen et au Bronze final, plusieurs enclos funéraires sont érigés. Durant l'Âge du Fer, se développent habitats et nécropoles. À partir du I ${ }^{\text {er }}$ siècle avant notre ère et jusqu'à la première moitié $d u v^{e}$ siècle de notre ère, l'espace est structuré par une villa, à proximité d'une importante voie romaine. Durant la période antique, un vaste réseau de fossés, connectés à au moins un collecteur principal (fig. 4e), est creusé pour drainer et matérialiser des limites parcellaires (Leprovost et Fleischer, 2014; Fleischer et Leprovost, 2015). Des traces de piétinements par des bestiaux ont été mises en évidence sur les bords du collecteur (P, fig. 4e).

Le réseau réticulé et ses digitations recoupent le substrat et les comblements des structures néolithiques et protohistoriques (incinération, fig. 4d) sur plus d'une soixantaine de centimères de profondeur (fig. 4b). Ils sont entaillés par les structures gallo-romaines (cave de la villa, fig. 4c).

\section{Seichamps (Meurthe-et-Moselle)}

À Seichamps (fouille F. Thiériot, 2016) le site archéologique mis au jour se situe au sein d'un petit versant drainé par un ruisseau temporaire. La fouille a permis de mettre au jour un paléochenal profond (fig. 1, site 13; fig. 5a) d'orientation globale sud-est/nord-ouest. Ce dernier semble recouper des fosses datées, par leur mobilier archéologique, du premier Âge du Fer (Hallstatt D3/La Tène ancienne). Selon les relations stratigraphiques et d'après les artefacts collectés dans la partie basale du comblement du chenal, la mise en place de ce dernier se serait faite entre la fin du $\mathrm{VI}^{\mathrm{e}}$ et le début du $\mathrm{v}^{\mathrm{e}}$ siècle avant notre ère. Dans la moitié sud et amont de l'emprise, ont également été observées plusieurs étapes dans d'aménagement permettant le franchissement du chenal encore humide et temporairement en eau (fig. 5d). Le premier état correspond à un aménagement en bois, fait de pieux et de planches, dont les datations radiocarbones donnent respectivement $23+/-10$ avant notre 


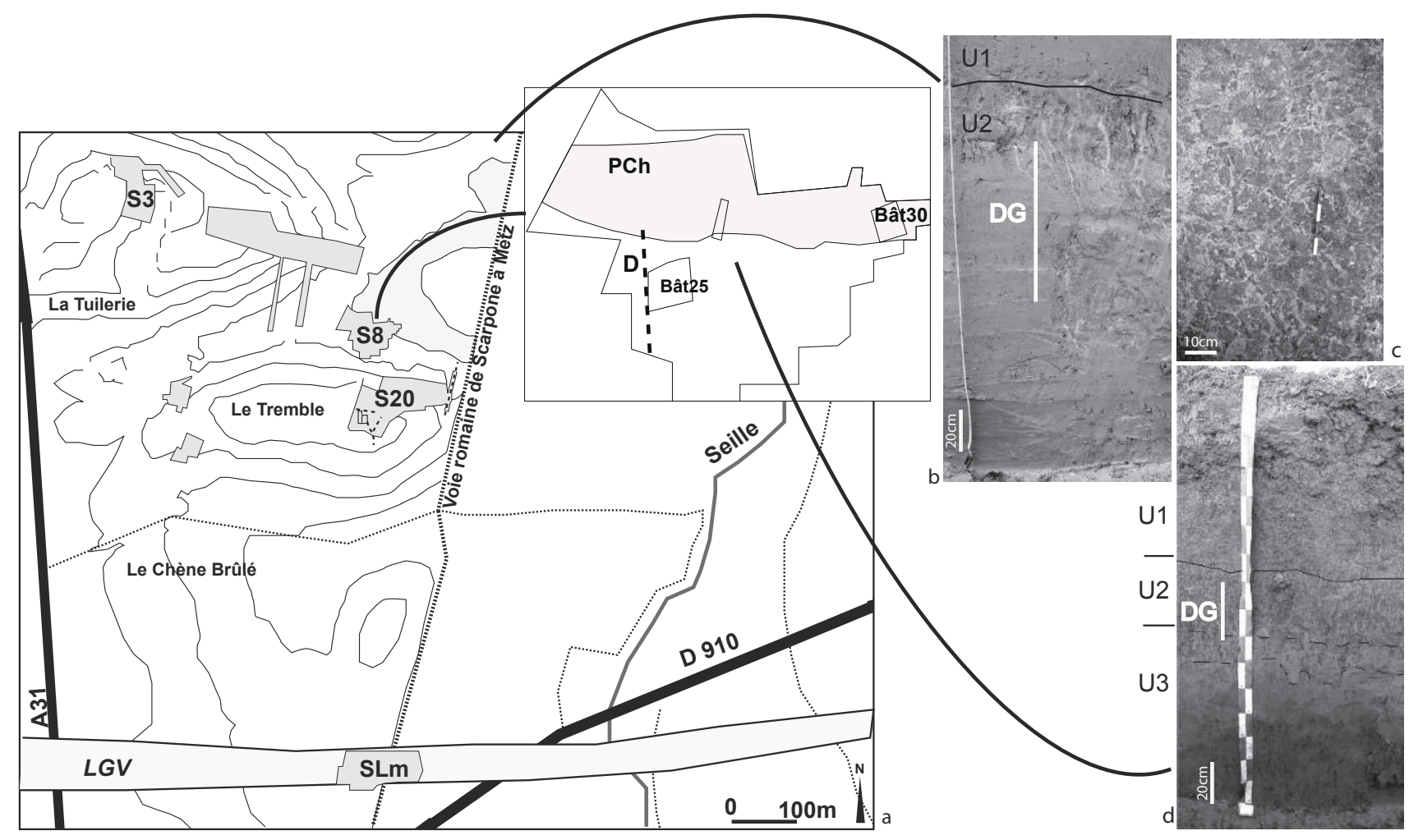

Figure 2 : Bouxières-sous-Froidemont (Moselle) a. localisation des différents sites (en gris). S8 : occupation de l'Âge du Bronze en berge du paléochenal (PCh, gris clair), S3; nécropole hallstattienne (tumulus) et gallo-romaine (incinérations) sur la colline, hors cote d'inondation, bât. 30 : construit sur poteau sur le chenal comblé à la fin de la première moitié du $\mathrm{I}^{\text {er }}$ siècle de notre ère, bât. 25 : habitat avec drainage (D) en limite de bâtiment occupé entre la seconde moitié du Ir siècle et la première moitié du II $^{\mathrm{e}}$ siècle de notre ère, S20 : ferme familiale occupée depuis le milieu du Ir siècle de notre ère jusqu'à la fin du Iv ${ }^{e}$ siècle avec de nombreux drains, SLm : relai de poste de Lesmesnils daté du début du I $^{\text {er }}$ siècle de notre ère, en bordure de la voie romaine de Scaponne à Metz, dans la zone inondable du lit majeur de la Seille, LGV : ligne ferroviaire grande vitesse, A31/D910 : autoroute/route départementale. b. séquence limono-argileuse non datée avec digitations (DG), observée sur la pente lors d'un diagnostic archéologique. c. vue en plan du réseau polygonal. d. U2 : limons argileux gris, mobilier laténien (5 avant notre ère/20 de notre ère) avec digitations (DG); U3 : limons argileux bruns oxydés (matériel protohistorique et planche de bois datée de l'Âge du Bronze final IIIa/b à la base); U1 : limons argileux gris, mouchetés, colluvionnés au cours de l'époque romaine (40-70 de notre ère).

Figure 2: Bouxières-sous-Froidemont (Moselle) a. location of the sites (dark grey). S8: Bronze Age occupation on the bank of a paleochannel (PCh, light grey), S3; cimetery with Hallstatt tumulus and Roman cremation on the hill out of the flooded area, bat. 30: building constructed on post on the channel filled in the end of the first half of the Ist century AD., batt. 25: house, with drains (D) around the walls, occupied between the second half of the Ist century and the first half of the IId century AD., S20: family farm occupied from the middle of the $1^{\text {st }}$ century AD to the end of the $4^{\text {th }}$ century, with numerous drains, SLm: post house of Lesmesnils, dated from beginning of the Ist century AD, along the Roman road from Scaponne to Metz, in the flood plain of the Seille River, LGV: fast train line. A31/D910: motor way/secondary road. b. undated siltyclay sequence with digitations (finger-like structures, DG) observed during a rescue archaeological diagnostic. c. upper view from the polygonal network. $\boldsymbol{d}$. U2: grey clayey loam, numerous artefacts (La Tène, -5 before the present era to AD 20) with digitations (DG); U3: brown loamy-clay with oxydation dots and protohistoric material (upper Bronze age IIIa/b); U: grey Roman colluvium with reddish oxidation dots (AD. 40-70).

ère et $8+/-10$ de notre ère. Par la suite, sont reconnus au moins trois niveaux de cailloutis, séparés par des colluvions limono-argileuses (fig. 5b). Ces empierrements successifs s'apparentent à un chemin qui aurait perduré pendant plusieurs siècles.
Sur ce site, le réseau polygonal a pu être observé au sein d'un horizon de colluvions limono-argileuses, d'environ 0,30 m d'épaisseur (fig. 5c, b), situé entre la première et la deuxième phase d'empierrement matérialisant le chemin (fig. 5b). Sa formation serait ici postérieure au tout début de notre ère. 


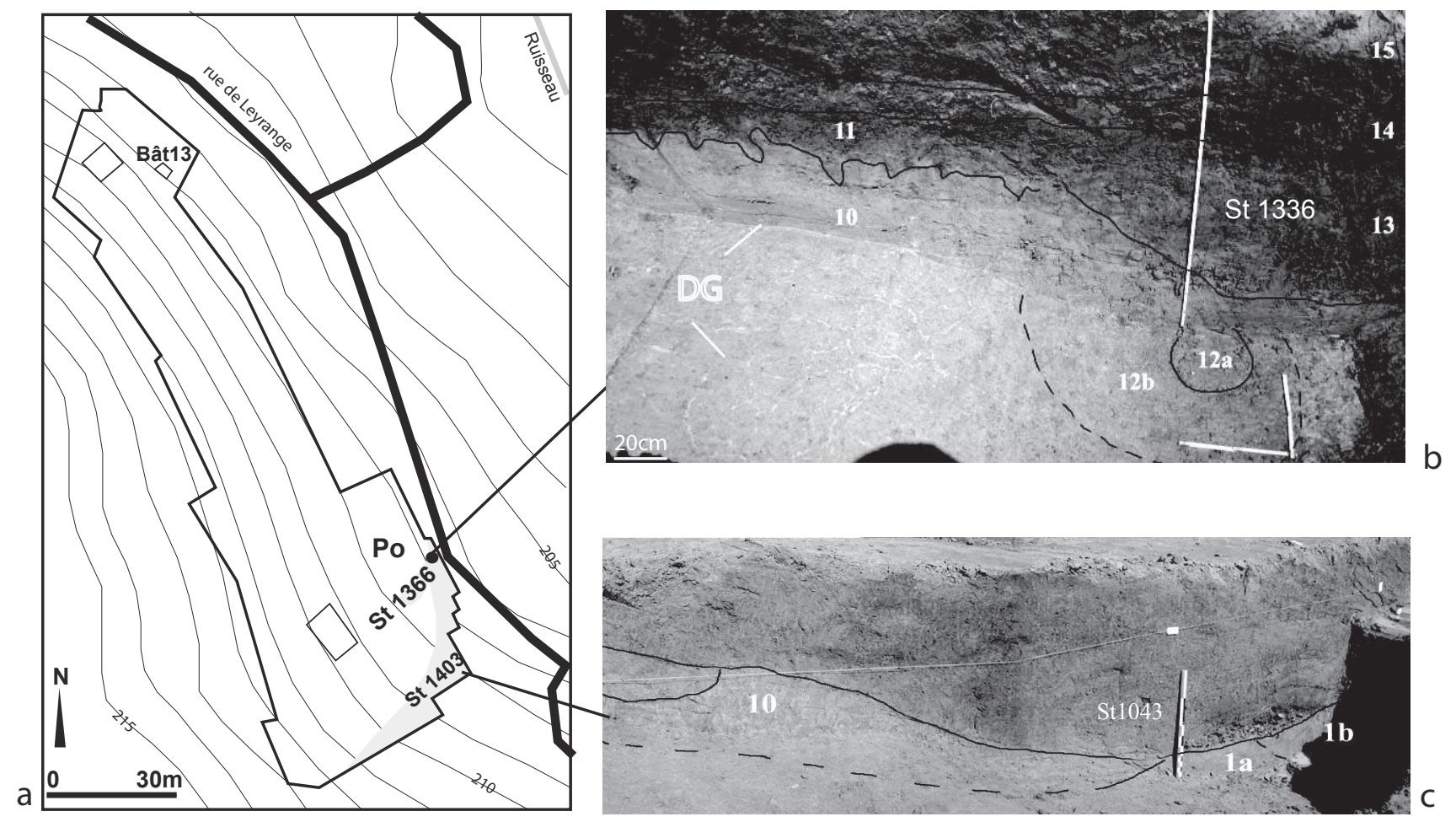

Figure 3 : Marspisch (Moselle) a. localisation de la zone étudiée (en gris foncé) sur la carte topographique. St1403: chemin creux principal, St1366 (13) : chemin creux secondaire abandonné au viI siècle, bât. 13 : fond de cabane du haut Moyen Âge (vi $/ \mathrm{VII}^{\mathrm{e}}$ s.), Po : poteau de bois daté de -56 avant notre ère. b. (10) : sédiment argilo-limoneux jaune dans lequel est enfoncé le poteau de bois (12a). (DG) digitations remplies de limon argileux blanchâtre. De forme polygonale réticulée en plan (10), elles prennent une forme concentrique (12b) suivant les traces de compaction du poteau (12a). (11) : limon brun-gris compact, peu bioturbé, fortement antropisé (micro-charbons, fragments millimétriques de briques/tuiles rouges), associé à l'occupation du haut Moyen Âge ; (St 1366) : remplissage limoneux brun-gris peu bioturbés, riches en micro-fragments de briques et tuiles rouges; (14) : limons colluvionnés récents, graveleux, noirs, non bioturbés, fortement anthropisés; (15) : limons colluvionnés récents, moins graveleux, nettement plus bioturbés; c. (St1043) : chemin creux principal recoupant l'horizon à digitations (10) et entaillant le substrat liasique (1a,1b).

Figure 3: Marspisch (Moselle) a. location of the excavated sites (dark grey) on the topographical map; St1403: main hollow way; St1366 (13): secondary hollow way abandoned during the VIIth century; bât. 13: early Middle-Age grubenhaus (VIth/VIIthc.); Po: wooden post dated 56 before the present era. $\boldsymbol{b}$. (10): yellow clayey-loamy sediment crossed by a wooden post (12a), with digitations filled in by a whitish clayey loam $(D G)$; The reticulated network (10) becomes concentric (12b) as a result of compaction created by the post (12a); (11): dark compact brown-grey loam slightly bioturbated, highly anthropogenized (micro-charcoals, rounded millimetric fragments of red tiles), associated to the early Medieval occupations; it overlies the lower sequence, and is cut by the (St1366): structure filled with non bioturbated brown-grey loam, strongly anthropogenized; (14): recent loamy colluvium, gritter black and non bioturbated at the base becoming less gritter and more bioturbated towards the top (15); $\boldsymbol{c}$. (St1043): main hollow lane cutting the digitated layer (10) down to the Lias substrate (1a, 1b).

\section{Caractéristiques générales communes aux sites présentés}

Sans être directement localisés dans le lit majeur d'un cours d'eau, les quatre sites étudiés sont tous positionnés en bas de pente en bordure de zone humide, sur une berge ou une ancienne terrasse. Le développement du réseau blanchâtre se fait dans un sédiment à dominante limono-argileuse, peu perméable. Les quatre sites détaillés précédemment sont pour l'instant les seuls à avoir permis le calage chronologique de ces formations blanchâtres dans la première moitié de l'Antiquité. Par ailleurs, associé à chacun de ces sites, existe un réseau de drainage plus ou moins important mais insuffisamment étudié à ce jour. Il s'agit généralement de fossés ouverts convergeant vers un collecteur artificiel ou une dépression naturelle. 




Figure 4 : Bernolsheim (Bas-Rhin) a. localisation des différentes structures et de la coupe étudiée sur la carte topographique. Vo : voie, Qa : qanat, Co : collecteur de drains, Vi : villa. b. (PC) Paléosol complexe pléistocène/holocène impacté par les digitations (DG) : (U5) sables roux rissiens avec quelques galets; (U4) limon-sableux brun clair, quelques biogaleries, illuviations tardiglaciaire/holocène en place, limite inférieure de développement du réseau polygonal; (U3) limon sableux brun-gris, structure polyédrique mieux marquée, moyennement bioturbé, érodé, mis en culture au cours de la protohistoire (fragments de céramiques, torchis), niveau d'apparition du réseau polygonal; (U2) : sable-limoneux brun-gris, structure particulaire, fortement bioturbé (biogaleries), nombreux galets, illuviations fragmentées remaniés, fragments de céramiques protohistoriques ou gallo-romains, torchis, colluvionné (colluvions historiques post-romaines, Col); (U1) : sables limoneux brun-roux, structure particulaire, abondants galets, fragments millimétriques de briques/tuiles roulés, illuviations remaniées, colluvionnées $(\mathrm{Col}) ;(\mathrm{R})$ : remblai végétal moderne. c. Cave antique au remplissage non affecté par les digitations. d. incinération de l'Âge du Fer traversée par des digitations (DG). e. Collecteur de drain avec traces de piétinement de bétail (P).

Figure 4: Bernolsheim (Bas-Rhin) a. general view of the site on the topographic map, with location of the different archaeological structures. Vo: road, Qa: qanat, Co: drain collector, Vi: villa. b. (PC): Pleistocene/Holocene complex palaeosol with digitations (DG): (U5): reddish Riss sands with some pebbles; (U4): light brown sandy loam slightly bioturbated with lateglacial/holocene undisturbed illuviations, lower development limit of the polygonal network; (U3) bioturbated brown-grey colluviated sandy loam with polyedric structure highly anthropogenized during protohistoric time (sherd, daub), upper limit of the polygonal network; (U2): brownish grey loamy highly bioturbated sand with pebbles, reworked illuviations, daub fragments, Iron Age/Roman sherds (post-Roman colluvium, Col); (U1): brown-grey loamy sand with numerous pebbles, highly anthropogenized (colluviated, Col); $(R)$ modern dumped organic sediment. $\boldsymbol{c}$. filling of a Roman cellar without cracks. $\boldsymbol{d}$. Iron Age cremation crossed by digitations (DG). e. Drain collector with cattle trampling traces (P). 


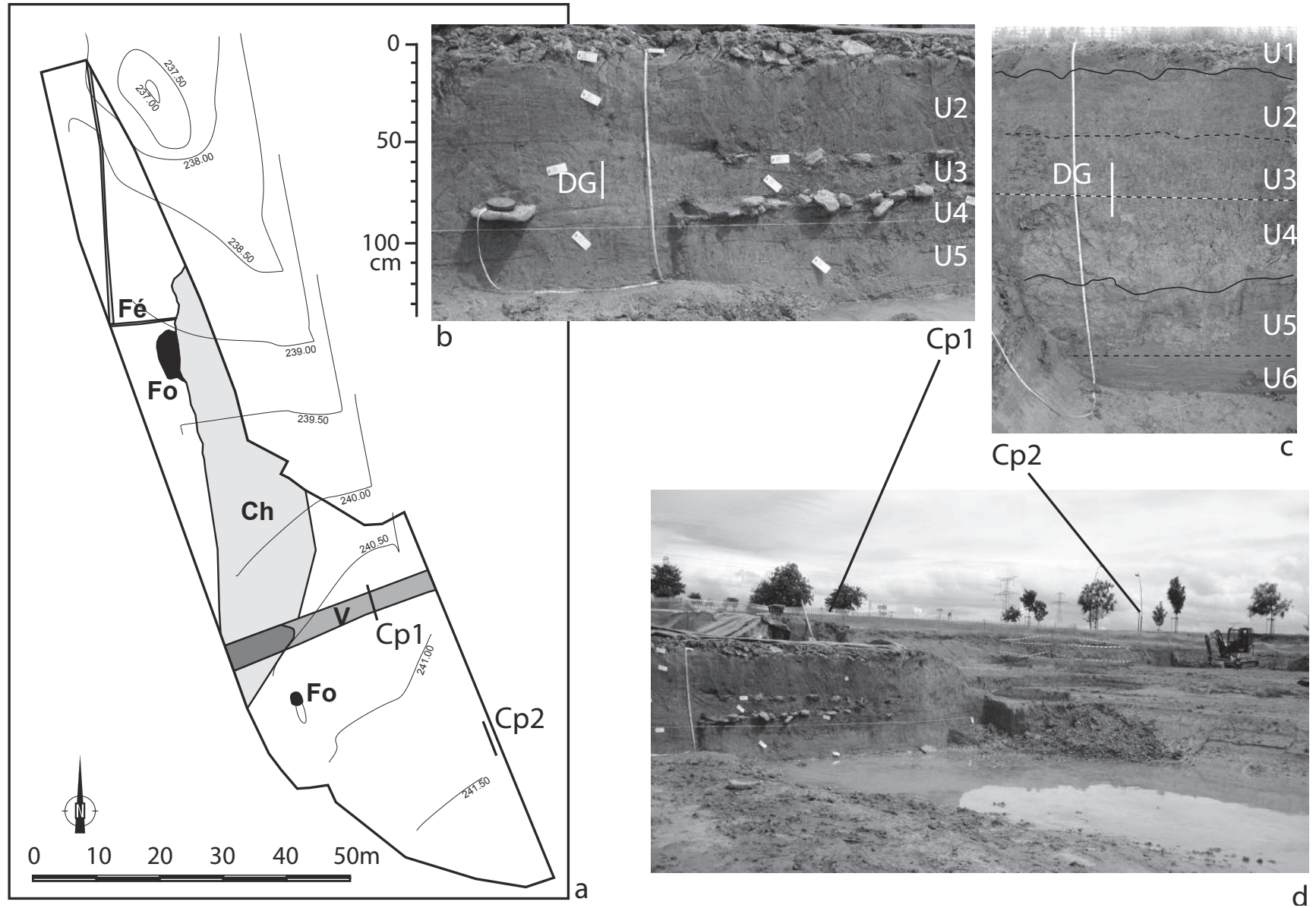

Figure 5 : Seichamps (Meurthe-et-Moselle) a. localisation sur la carte topographique des différentes structures et des coupes étudiées (Cp) ; Fé : fossé; Fo : fosses du Ir Âge du Fer; Ch : chenal; V : voie. b. Cp1, les trois états d'empierrement de la voie avec la position des digitations (DG). c. Cp2, (U6) : marne en place oxydée en surface; (U5) : marne brune, poches de sables oxydés; (U4) : marne sableuse brun-gris, bioturbée, concrétions et imprégnations de fer et manganèse; (U3) : argile sablo-limoneuse brune mouchetée avec digitations (DG); (U2) : limon sablo-argileux brun-jaune, structure polyèdrique subangulaire, très bioturbé, fragments roulés millimétriques de céramique;(U1) : limon brun-gris bioturbé, structure grumeleuse. d. vue générale du site avec positionnement des coupes 1 et 2 , sous la lame d'eau le premier état de la voie : planches $(8+/-10$ de notre ère $)$ et pieux $(23+/-10$ avant notre ère).

Figure 5: Seichamps (Meurthe-et-Moselle) a. location of the structures and studied profiles (Cp) on the topographic map; Fé: ditch, Fo: pit from the 1st Iron Age; Ch: channel; V: road. b. Cp1, profile showing the three states of the road metalling with the position of the digitations (DG). c. Cp2, (U6): in situ oxidized marl; (U5): brown marl, oxidized sand pockets; (U4): brown-grey sandy bioturbated marl, iron/manganese concretions and impregnations; (U3): brown sandy-loamy clay with reddisch dots, lower development limit of the digitations (DG); (U2): yellow-brown sandy-clayey loam, polyedric subangular structure, strongly bioturbated, rolled millimetric sherds fragments; (U1): brown-grey loam with bioturbated crumby structure. $\boldsymbol{d}$. general picture of the site with the position of profiles 1 and 2; under the water, first pavement of the road maid of wooden planks $(A D 8+1-10)$ and posts $(23+1-10$ before the present era $)$.

\section{Description des formes polygonales digitées}

En plan, à son niveau d'apparition, le maillage est polygonal (connexion des côtés en Y) et de faible diamètre (10$20 \mathrm{~cm}$ en surface). En profondeur, son diamètre augmente jusqu'à atteindre une quarantaine de centimètres. En coupe les digitations, très nombreuses au sommet du niveau, s'enfoncent dans le substrat de 20 à $30 \mathrm{~cm}$, bien que certaines puissent descendre jusqu'à $1 \mathrm{~m}$ de profondeur. À Bouxièressous-Froidemont, elles traversent un fragment de céramique laissé à plat dans l'horizon du Bronze final U3 (fig. 2d). Les digitations sont caractérisées par un sédiment légèrement moins argileux que l'encaissant. Elles sont blanchâtres car appauvries en fer et souvent soulignées d'un liseré rougeâtre sur les bords. On y observe des éléments organiques et des petits charbons (C, fig. 6a) bien que l'on retrouve ces der- 


niers également en dehors des zones blanchies. Soulignée par une forte ferruginisation de l'encaissant (fig. 6f), la limite entre la zone blanchie et l'encaissant est nette à l'œil nu (fig. 6a, d, g) et au microscope (fig. 6d, i). La granulométrie des digitations montre peu de différence avec l'encaissant (fig. 6e, f). Observées en lames minces, les illuviations d'argile limpide sont plus nombreuses et mieux visibles dans l'ensemble du sédiment que dans les zones blanchies (fig. 6c). En bordure de ces zones, apparaissent de fines fissures verticales (fig. 6f) soulignées par des illuviations jaunes limpides (fig. 6c, i). Un fragment d'ancien BT de luvisol (Baize et Girard, 1992) remanié et traversé par une zone blanchie (fig. 6b) a également été observé.

L'inventaire des digitations polygonales repérées à ce jour en contexte de diagnostic dans le Grand Est (fig. 7) montre que loin d'être anecdotiques, ces traits pédologiques se retrouvent déjà sur au moins 14 sites à l'échelle régionale. Hormis les 4 sites détaillés ci-dessus, ce tableau montre 3 sites où les digitations sont postérieures à la protohistoire et un site où elles sont postérieures au Néolithique.

\section{INTERPRÉTATION}

Dans ce chapitre, nous allons passer en revue les différents mécanismes permettant la formation de digitations associées à un réseau polygonal. Par déduction, nous pourrons ensuite proposer une hypothèse sur le mode de formation de telles figures au début de l'Antiquité dans le Grand Est de la France.

\section{Origine biologique?}

L'orientation verticale en coupe, la grande profondeur et la faible largeur de ces structures peut faire penser à des galeries de lombrics reprises ou non par des racines. Mais leur agencement polygonal en surface élimine toute origine biologique.

\section{Origine périglaciaire?}

L'agencement polygonal est généralement lié à l'ouverture de fentes lors de la contraction thermique des sols par le gel en contexte périglaciaire. Des polygones sont alors de taille plurimétrique et souvent visibles en photo aériennes (Bertran et al., 2017; Andrieux et al., 2016). Les fentes (pseudomorphoses de coin de glace) associées sont de largeur pluri-décimétrique, peuvent atteindre $3 \mathrm{~m}$ de profondeur et leur remplissage est hétérogène. Le sédiment encaissant peut être affecté par le gel selon sa composition granulométrique.
Des petits polygones de diamètre décimétrique et peu profonds, plutôt liés à la dessiccation, subdivisent souvent ces grands polygones. Dans les sédiments limono-argileux le compactage va générer un fragipan imperméable où, par la suite, seul le remplissage moins dense des fentes va autoriser le passage des racines en profondeur (Van Vliet-Lanoë et Langohr, 1981; Langohr, 2010). D'autres traits typiques sont observés comme une structure lamellaire du sédiment, la position verticale des cailloux, des cheminées d'involutions (Van Vliet-Lanoë et al., 1984; Bertran et al., 2017).

Aucun de ces faits n'ont été observés associés aux réseaux polygonaux décrits dans le cadre de cette étude. Par ailleurs, le calage chronologique des traits décrits dans cet article au début de notre ère interdit toute hypothèse de formation périglaciaire anté-Holocène.

\section{Hypothèse proposée : des réseaux de fentes de dessiccation}

Peu de travaux concernent les conditions de fonctionnement des fentes de dessiccation actuelles en contexte tempéré, mis à part quelques-uns sur la description physique et mécanique de l'évolution des fentes dans des sols très argileux (Reeve et al., 1980; Kishné, 2010), en lien avec les désordres créés par le retrait/gonflement sur le bâti (Blight, 2009; Weinberger, 1999; Peron et al., 2009, 2012). Après plusieurs cycles de stress hydrique expérimental d'une couche de bentonite de $3 \mathrm{~cm}$ d'épaisseur, les fentes - au contour initialement anguleux en surface - réapparaissent toujours au même endroit en s'arrondissant, passant d'une jonction en $T$ à une jonction en $\mathrm{Y}$ entre les bords des polygones (Goehring et al., 2010). Des oscillations courtes de la teneur en eau journalière du sol associées à un déficit annuel semblent suffire à déclencher une dessiccation des sols (Kishné et al., 2010). Ce type de petites fentes peut être observé localement dans les flaques en cours d'assèchement ou au sein de sols argileux dans des zones à forte sécheresse estivale (fig. 8). Quelques travaux relatent également de fentes liées à de longues sécheresses dans les salars (Risacher, 1992) et sols désertiques (Delhoume et al., 1988) des régions arides. Mais globalement on trouve peu d'observations sur les températures et l'hygrométrie pouvant renseigner sur les conditions climatiques d'apparition de ces fentes sous nos climats tempérés. Toutefois, un rapport québécois précise que pour voir l'apparition de fentes dans un sédiment argilo-limoneux il faut au moins $35 \%$ d'argiles, une baisse conséquente de la nappe hydrique, un fort déficit estival en eau météorique (inférieur à $150 \mathrm{~mm}$ en juillet-août), une couverture neigeuse faible qui accentuerait les cycles de gel/dégel en surface et un sol non à peu couvert (culture de céréales ou maïs par 


\begin{tabular}{|c|c|c|c|c|c|c|c|c|c|c|}
\hline Site & Cadre étude & Topographie & Sédiment & $\begin{array}{l}\text { Position stratigra- } \\
\text { phique du niveau } \\
\text { affecté }\end{array}$ & \begin{tabular}{|l|} 
Profon- \\
deur des \\
fentes
\end{tabular} & $\begin{array}{l}\text { Calage } \\
\text { chronologique }\end{array}$ & \begin{tabular}{|l} 
Archéologie, \\
drains, parcel- \\
laire
\end{tabular} & $\begin{array}{l}\text { Forme, } \\
\text { maillage }\end{array}$ & Remarques & Référence \\
\hline $\begin{array}{l}\text { Ancerville } \\
\text { (Meuse) } \\
\text { Fig. } 1,4\end{array}$ & $\begin{array}{l}\text { Diag. INRAP } \\
\text { Fig. } 1,4\end{array}$ & Lit majeur & $\begin{array}{l}\text { Limons } \\
\text { argileux }\end{array}$ & $\begin{array}{l}\text { Sous } 30 \mathrm{~cm} \\
\text { d'horizon de labour }\end{array}$ & $70 \mathrm{~cm}$ & 1 & 1 & $\begin{array}{l}\mathrm{Y} \\
10-20 \mathrm{~cm}\end{array}$ & 1 & Rachet et Gebhardt, 2012 \\
\hline $\begin{array}{l}\text { Bernolsheim } \\
(67) \\
\text { Fig. } 1,17\end{array}$ & Fouille AA & $\begin{array}{l}\text { Terrasse } \\
\text { alluviale }\end{array}$ & \begin{tabular}{|l} 
Sables \\
argileux \\
et limons \\
argileux
\end{tabular} & \begin{tabular}{|l} 
Sous $80 \mathrm{~cm}$ de col- \\
luvions historiques
\end{tabular} & $70 \mathrm{~cm}$ & $\begin{array}{l}\text { Entre l'Âge du Fer } \\
\text { et l'Antiquité }\end{array}$ & $\begin{array}{l}\text { Villa, réseau de } \\
\text { fossés, collecteur } \\
\text { antiques }\end{array}$ & $\begin{array}{l}Y \\
20-30 \mathrm{~cm}\end{array}$ & 1 & $\begin{array}{l}\text { Fleischer } \text { et al., } 2011 \\
\text { Leprovost/Fleischer, } 2014 \\
\text { Leprovost/Fleischer, } 2015\end{array}$ \\
\hline $\begin{array}{l}\text { Bouxières-sous- } \\
\text { Froidemont (57) } \\
\text { Fig. } 1,8\end{array}$ & $\begin{array}{l}\text { Fouille INRAP } \\
\text { Fig. } 1,8\end{array}$ & $\begin{array}{l}\text { Bas de pente, } \\
\text { fond de vallon }\end{array}$ & $\begin{array}{l}\text { Limons } \\
\text { argileux }\end{array}$ & $\begin{array}{l}\text { Sous } 30 \mathrm{~cm} \\
\text { d'horizon de labour }\end{array}$ & $100 \mathrm{~cm}$ & $\begin{array}{l}\text { Entre la Tène finale } \\
\text { et le début de } \\
\text { l'Antiquité }\end{array}$ & $\begin{array}{l}\text { Fossé de drai- } \\
\text { nage antique }\end{array}$ & $\begin{array}{l}Y \\
10-20 \mathrm{~cm}\end{array}$ & 1 & $\begin{array}{l}\text { Defferessigne et al., } 2012 \\
\text { Dohr } \text { et al., } 2014\end{array}$ \\
\hline $\begin{array}{l}\text { Cerville (54) } \\
\text { Fig. } 1,14\end{array}$ & $\begin{array}{l}\text { Diag. INRAP } \\
\text { Fig. } 1,10\end{array}$ & $\begin{array}{l}\text { Bas de pente, } \\
\text { fond de vallon }\end{array}$ & $\begin{array}{l}\text { Limons } \\
\text { argileux }\end{array}$ & \begin{tabular}{|l|} 
Sous $30 \mathrm{~cm}$ \\
d'horizon de labour
\end{tabular} & $40 \mathrm{~cm}$ & 1 & 1 & $\begin{array}{l}\mathrm{Y} \\
10-20 \mathrm{~cm}\end{array}$ & 1 & Jeandemange, 2006 \\
\hline $\begin{array}{l}\text { Chamagne } \\
\text { Fig. 1, } 19\end{array}$ & Diag. INRAP & $\begin{array}{l}\text { Haute terrasse } \\
\text { de la Moselle }\end{array}$ & $\begin{array}{l}\text { Limons } \\
\text { argileux }\end{array}$ & $\begin{array}{l}\text { Sous } 30 \mathrm{~cm} \\
\text { d'horizon de labour }\end{array}$ & $100 \mathrm{~cm}$ & $\begin{array}{l}\text { Postérieur à la } \\
\text { Protohistoire }\end{array}$ & $\begin{array}{l}\text { Fossés protohis- } \\
\text { toriques } \\
\text { et non datés, } \\
\text { drains modernes }\end{array}$ & $\begin{array}{l}\mathrm{Y} \\
10-20 \mathrm{~cm}\end{array}$ & $\begin{array}{l}\text { Affecte clairement le } \\
\text { remplissage du fossé } \\
\text { protohistorique }\end{array}$ & Rachet et al., 2017 \\
\hline $\begin{array}{l}\text { Coussey (88) } \\
\text { Fig. } 1,18\end{array}$ & Diag. INRAP & \begin{tabular}{|l|} 
Berge Salmo- \\
nelle/Meuse
\end{tabular} & $\begin{array}{l}\text { Limons } \\
\text { argileux }\end{array}$ & \begin{tabular}{|l|} 
Sous $60 \mathrm{~cm}$ de col- \\
luvions historiques
\end{tabular} & $30 \mathrm{~cm}$ & $\begin{array}{l}\text { Contemporain aux } \\
\text { niveaux antiques }\end{array}$ & 1 & $\begin{array}{l}\text { Pas vu en } \\
\text { plan }\end{array}$ & 1 & Meyer et al., 2015 \\
\hline $\begin{array}{l}\text { Illange (57) } \\
\text { Fig. } 1,1\end{array}$ & Diag. INRAP & $\begin{array}{l}\text { Berge de la } \\
\text { Moselle }\end{array}$ & $\begin{array}{l}\text { Limons } \\
\text { argileux }\end{array}$ & \begin{tabular}{|l} 
Sous $60 \mathrm{~cm}$ de col- \\
luvions historiques
\end{tabular} & $30 \mathrm{~cm}$ & \begin{tabular}{|l|} 
Postérieur aux \\
colluvions de l'Âge \\
du Fer
\end{tabular} & 1 & $\begin{array}{l}\text { Pas vu en } \\
\text { plan }\end{array}$ & 1 & Gebhardt, 2004 \\
\hline $\begin{array}{l}\text { La Salle (88) } \\
\text { Fig. } 15\end{array}$ & Diag. INRAP & $\begin{array}{l}\text { Alluvions du } \\
\text { Neuné }\end{array}$ & $\begin{array}{l}\text { Limons } \\
\text { argileux }\end{array}$ & $\begin{array}{l}\text { Sous } 30 \mathrm{~cm} \\
\text { d'horizon de labour }\end{array}$ & $40 \mathrm{~cm}$ & 1 & 1 & $\begin{array}{l}\mathrm{Y} \\
20-30 \mathrm{~cm}\end{array}$ & 1 & Rachet et Gebhardt, 2010 \\
\hline $\begin{array}{l}\text { Marspisch (57) } \\
\text { Fig. } 1,3\end{array}$ & Fouille INRAP & $\begin{array}{l}\text { Pente, } \\
\text { fond de vallon }\end{array}$ & $\begin{array}{l}\text { Limons } \\
\text { argileux }\end{array}$ & $\begin{array}{l}\text { Sous } 110 \mathrm{~cm} \mathrm{de} \\
\text { colluvions histo- } \\
\text { riques }\end{array}$ & $>80 \mathrm{~cm}$ & $\begin{array}{l}\text { Entre -56 avant } \\
\text { notre ère et la } \\
\text { période mérovi- } \\
\text { gienne }\end{array}$ & Chemin creux & $\begin{array}{l}\mathrm{Y} \\
20 \mathrm{~cm}\end{array}$ & $\begin{array}{l}\text { S'oriente de façon } \\
\text { concentrique selon } \\
\text { un trou de poteau }\end{array}$ & Mondi et al., 2015 \\
\hline $\begin{array}{l}\text { Moncel les } \\
\text { Lunévilles (54) } \\
\text { Fig. } 1,10\end{array}$ & Diag INRAP & $\begin{array}{l}\text { Lit majeur de } \\
\text { la Meurthe }\end{array}$ & $\begin{array}{l}\text { Limons } \\
\text { argileux }\end{array}$ & $\begin{array}{l}\text { Sous } 30 \mathrm{~cm} \\
\text { d'horizon de labour }\end{array}$ & $60 \mathrm{~cm}$ & 1 & 1 & $\begin{array}{l}Y \\
20-30 \mathrm{~cm}\end{array}$ & $\begin{array}{l}\text { 'oriente selon des } \\
\text { fentes périglaciaires } \\
\text { en profondeur }\end{array}$ & Forelle et Gebhardt, 2012 \\
\hline $\begin{array}{l}\text { Phalsbourg (57) } \\
\text { Fig. } 1,12\end{array}$ & Diag INRAP & \begin{tabular}{|l} 
Bord de \\
plateau limite \\
zone humide
\end{tabular} & $\begin{array}{l}\text { Limons } \\
\text { argileux }\end{array}$ & $\begin{array}{l}\text { Sous } 100 \mathrm{~cm} \mathrm{de} \\
\text { colluvions histo- } \\
\text { riques }\end{array}$ & $60 \mathrm{~cm}$ & 1 & 1 & $\begin{array}{l}Y \\
20-30 \mathrm{~cm}\end{array}$ & 1 & Jeandemange et al., 2013 \\
\hline \multirow{3}{*}{$\begin{array}{l}\text { Remomeix (88) } \\
\text { Fig. } 1,16\end{array}$} & \multirow{3}{*}{ Diag INRAP } & \multirow{3}{*}{$\begin{array}{l}\text { Lit majeur de } \\
\text { la Fave }\end{array}$} & \multirow{3}{*}{$\begin{array}{l}\text { Limons } \\
\text { argileux }\end{array}$} & \multirow{3}{*}{$\begin{array}{l}\text { Sous } 30 \mathrm{~cm} \\
\text { d'horizon de labour }\end{array}$} & \multirow{3}{*}{$40 \mathrm{~cm}$} & \multirow{3}{*}{1} & \multirow{3}{*}{$\begin{array}{l}\text { Billon et canaux } \\
\text { d'irrigation } \\
\text { modernes }\end{array}$} & \multirow{3}{*}{$\begin{array}{l}\mathrm{Y} \\
20-30 \mathrm{~cm}\end{array}$} & \multirow{3}{*}{1} & Jude et Gebhardt, 2006, \\
\hline & & & & & & & & & & $\begin{array}{l}\text { Gücker et Gebhardt, } \\
2007\end{array}$ \\
\hline & & & & & & & & & & Dohr et Gebhardt, 2008 \\
\hline $\begin{array}{l}\text { Seichamps (54) } \\
\text { Fig. } 1,13\end{array}$ & Fouille INRAP & $\begin{array}{l}\text { Bord de paléo- } \\
\text { vallon }\end{array}$ & $\begin{array}{l}\text { Limons } \\
\text { argileux }\end{array}$ & \begin{tabular}{|l|} 
Sous $60 \mathrm{~cm}$ de col- \\
luvions historiques
\end{tabular} & $40 \mathrm{~cm}$ & \begin{tabular}{|l|}
23 avant notre ère \\
18 de notre ère Ante \\
Quem \\
\end{tabular} & I & Y & 1 & $\begin{array}{l}\text { Thiériot } e t \text { al., étude en } \\
\text { cours }\end{array}$ \\
\hline $\begin{array}{l}\text { Terville (57) } \\
\text { Fig. } 1,3\end{array}$ & Fouille INRAP & \begin{tabular}{|l} 
Bord de lit \\
majeur de la \\
Moselle
\end{tabular} & $\begin{array}{l}\text { Limons } \\
\text { sableux }\end{array}$ & $\begin{array}{l}\text { Sous } 80 \mathrm{~cm} \text { d'allu- } \\
\text { vions limoneux }\end{array}$ & $40 \mathrm{~cm}$ & $\begin{array}{l}\text { /Postérieur au } \\
\text { Néolithique }\end{array}$ & 1 & $\begin{array}{l}Y \\
20-30 \mathrm{~cm}\end{array}$ & 1 & Petitdidier, 2018 \\
\hline
\end{tabular}

Figure 7 : tableau des caractéristiques des réseau polygonaux et digitations, observés au cours des fouilles et des diagnostics archéologiques.

Figure 7: table of polygonal networks and digitations caracteristics observed during archeological rescue operations. 

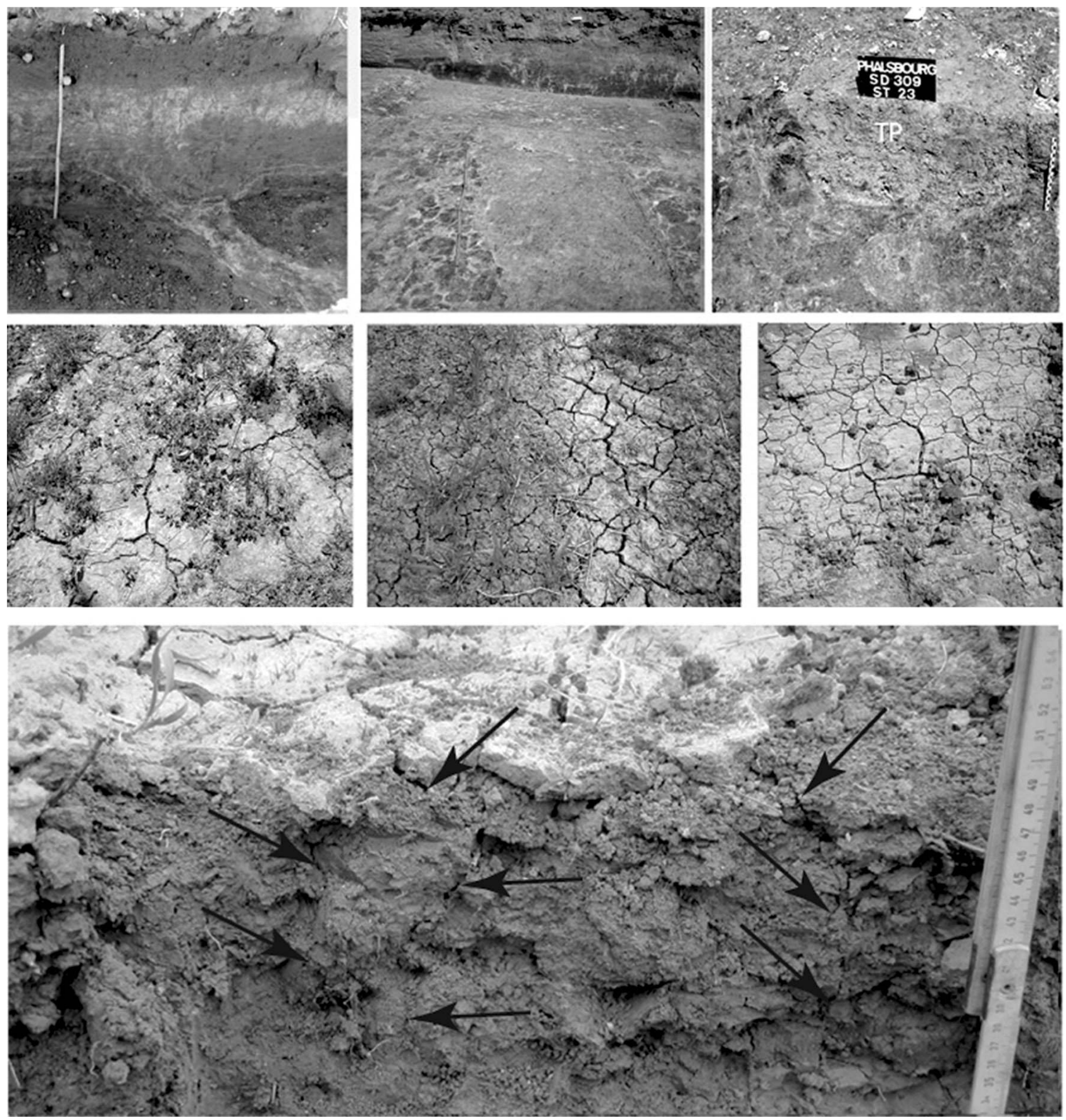

Figure 8 : de haut en bas et de gauche à droite : Moncel-les-Lunéville (Meurthe-et-Moselle) coin de glace quaternaire jouant un rôle de drain prédéterminant l'orientation des digitations blanchâtres. Chamagne (Vosges) : digitations blanchâtre postérieures à un fossé protohistorique. Phalsbourg (Moselle) : trou de poteau (TP) non daté postérieur au développement des digitations. Pise (Italie) : réseau de dessiccation polygonal actuel dans un jardin public, Sarrebourg (Moselle) : formation d'un réseau de fentes polygonales en Y, dans un champ sous le passage d'une roue d'engin agricole, Marspich (Moselle) craquelure des argiles en T sur une surface archéologique décapée, Achenheim (Alsace) : Fentes de dessiccation actuelles (plan et coupe) dans le loess.

Figure 8: from top to bottom and left to right: Moncel-les-Lunéville (Meurthe-et-Moselle), Quaternary ice wedge wich promoted the drainage and the location, shape and orientation of the bleached digitations. Chamagne (Vosges): bleached digitations younger than the proto-historical ditch. Phalsbourg (Moselle): undated post-hole (TP) cutting the digitations. Pisa (Italy): present polygonal desiccation network in a public garden. Sarrebourg (Moselle): Y shaped polygonal cracks under the compacted track of an agricultural machinary. Marspich (Moselle); T shaped cracks on a puddle surface. Achenheim (Alsace): actual desiccation cracks (plane and profile) in loess. 
exemple). Les fentes de retrait peuvent alors atteindre 75 à $100 \mathrm{~cm}$ de profondeur (Bégin, 2007).

Dans une note de la Revue de géographie de Lyon, Georges Plaisance (1954) fait état de " veines blanchâtres délimitant en surface des polyèdres plus ou moins denses dans des horizons profonds de limons ". Une discussion avec ses pairs les associe à des fentes minces et profondes de dessiccation que vient remplir une eau chargée en matières humiques circulant dans ces zones privilégiées avant réduction du fer et du manganèse. Des glosses d'origine périglaciaire, affectant des sols argileux sur terrasses gravelo-sableuses drainantes anciennes (sols lessivés glossiques des zones tempérées atlantiques; Duchaufour, 1983), sont également décrites comme des fentes de retrait remplies d'argiles et de fer lessivés où l'eau acide circulant va décolorer et dégrader les argiles fines.

Plus récemment un article norvégien (Sauer et al., 2013) décrit des langues blanchâtres dans les sols (albeluvic; IUSS Working Group WRB, 2015) et les associe à des fentes polygonales de dessiccation liées au ressuyage des sols après une remonté isostatique du continent par rapport au niveau marin. Cette première étude d'une chrono-séquence d'albeluvisols (IUSS Working Group WRB, 2015), ou luvisols dégradés (Baize et Girard, 1992; Baize et Jamagne, 2009), développée sur limons marins, montre que des fentes polygonales profondes de dessiccation se forment dans ces sols dès leur ressuyage et leur compaction (Sauer et al., 2013). Sauer et ses collègues pointent la rapide décarbonatation du sédiment liée aux acides dégagés dans le sol par l'oxydation du fer le long de la porosité. Selon Roger Langohr (com. pers.), l'eau percole de préférence le long de ces mini-fissures et les racines vont y trouver un chemin direct vers la profondeur. Lorsque ces dernières meurent, et que la fente se colmate, la matière organique entraîne une nouvelle réduction du fer et du manganèse qui colore en gris l'intérieur du remplissage. Enfin, les parois de ces fentes sont soulignées par une auréole d'oxydation brun-rouge, liée à la migration $\mathrm{du}$ fer et du manganèse lors d'alternances de phases de forte sécheresse et d'humidité (Roger Langohr, com. pers). Mais les formes de digitation peuvent apparaître en profondeur, en l'absence de fentes de retrait en surface, dans un sol non forcément saturé en eau dont la texture fine repose sur un sédiment de texture plus grossière (Bégin, 2007).

La dégradation des luvisols ferait donc suite au réssuyage d'un sol argilo-limoneux. Pour Denis Baize (2008), elle démarrerait préférentiellement le long de grosses fissures verticales colmatées (par exemple des fentes en coin périglaciaires - fig. 6 - ou de sécheresse) donnant naissance à des " glosses " qui grandissent pour finir en une dégradation beaucoup plus "diffuse " de l'horizon BTg. Ces langues doivent être plus profondes que larges $(5$ à $15 \mathrm{~mm}$ selon la proportion de sable), présenter une occupation du volume de plus de $15 \%$ de l'horizon et correspondre à des concentrations relativement continues d'éléments du squelette (Baize et Girard, 1992; Lozet et Mathieu, 1997). Mais ce n'est pas un phénomène continu (Baize, 2008; Baize et Girard, 2009). La position des luvisols dégradés dans la catena (Jamagne, 1973; Baize, 2008) souligne leur occurrence préférentielle en bordure de zone humide plus rapidement asséchée lors d'une baisse de la nappe phréatique. Sur le plateau lorrain de nombreux sols se développent au sein de séquences limoneuses peu épaisses déposées sur un substratum argilo-marneux. Pendant la saison humide la saturation en argile de horizon BT favorise la formation d'une nappe perchée générant une forte oxydation de ce niveau, qui sera ensuite traversé par des glosses issues de l'horizon E albique au cours de la dégradation du luvisol (Caillier, 1977; Gury, 1990; Florentin, 2005; Jamagne 2011). La même configuration se retrouvera sur les moyennes terrasses du bassin de la Moselle, où un fragipan périglaciaire imperméable (Van Vliet-Lanoë et Langohr, 1981) pourrait favoriser une stagnation hydrique (Caillier, 1977; Gury, 1990).

La dégradation naturelle des luvisols est considérée comme un phénomène pouvant s'étaler de quelques milliers à une dizaine de milliers d'années (Baize, 2008; Sauer et al., 2013). Or ce phénomène semble nettement plus rapide dans les parcelles drainées pour réguler l'engorgement saisonnier en eau. Dans sa thèse sur l'influence du drainage agricole par tuyaux enterrés sur les sols limoneux de l'Yonne, David Montagne (2006) montre qu'en modifiant les flux d'eau et le $\mathrm{pH}$ du sol, le drainage et l'amendement transforment les conditions d'oxydo-réduction du sol et favorisent ou ralentissent la dégradation des sols. Cette dégradation augmente à proximité des drains et peut mener à une dégradation visible en une quinzaine d'années. Ainsi, la mise en culture semble bien capable d'accélérer la dégradation morphologique des sols par l'implantation d'un réseau de drainage enterré ou de la ralentir par le chaulage.

\section{Discussion}

Dans les quatre cas étudiés dans cet article, le réseau de dessiccation semble prendre forme au début de notre ère dans des formations mises en place au cours de la Protohistoire ou au tout début de l'époque gallo-romaine. La particularité commune observée est le positionnement de tous ces réseaux en pied de pente, en limite de la plaine alluviale active à l'époque romaine. Ils se développent dans un sédiment limono-argileux assez bien drainé mais où le toit de la nappe phréatique actuelle est encore, ou de nou- 
veau, assez proche de la surface. Ces traits semblent donc liés à une dessiccation du sol. En effet, une baisse du niveau de la nappe phréatique ou perchée va assécher le sédiment limono-argileux et entraîner la dégradation du luvisol. Il reste à discuter de l'origine de cette baisse du niveau de la nappe. Deux hypothèses, non exclusives, peuvent être avancées : une période de sécheresse climatique et/ou un assèchement volontaire du milieu par drainage agricole au début du premier millénaire.

Les précédents travaux ont montré qu'en Lorraine, les populations romanisées ont dû composer avec des sols appauvris et des paysages largement transformés par leurs prédécesseurs gaulois (Gebhardt et al., 2014). Cette période coïncide aussi avec l'amélioration climatique du petit optimum romain (POR), maintenant bien reconnu régionalement (Tegel et Hakelberg, 2017). Au cours de cet événement climatique stable, des déficits hydriques pourraient avoir été assez importants pour provoquer, dans les sols limono-argileux mal drainés, la formation de fentes de retrait caractéristiques, voire la fissuration des revêtements argileux (Gebhardt et al., 2014) et un assèchement rapide, au moins saisonnier, permettant l'exploitation de terres jusque-là très humides (Solgnes, Gebhardt, 2005). De nombreux travaux régionaux et extra-régionaux semblent aller dans le sens d'années à fort stress hydrique au cours de la haute antiquité dans le Grand Est. Pour McCormick et al. (2011), le début de l'Antiquité est une période climatique stable ponctuée de fortes sécheresses et de grosses inondations. Faisant suite à l'âge glaciaire du fer (AGF) plus frais et humide, le tournant de notre ère est aussi une phase de régression des glaciers alpins (Holzhauser et al., 2005), de faible charge pour la Saône et le Rhône (Bravard, 1997) et de baisse du niveau des lacs du Jura (Van Geel et Magny, 2002). Une courbe d'anomalies thermiques et pluviales, établie sur des données dendrochronologiques ouest européennes, montre des pluies printanières faibles, corrélées avec des pics de forte température estivale dans la première moitié du $\mathrm{I}^{\mathrm{er}}$ siècle de notre ère (Büntgen et al., 2011).

Durant cette période, de nombreux témoignages archéologiques soulignent une baisse du niveau des nappes hydriques comme dans le Grand-Marais du Jura suisse (Schwab, 2003) où il a pu descendre jusqu'à $1 \mathrm{~m}$ sous la cote actuelle. C'est aussi dans le courant du $\mathrm{I}^{\text {er }}$ siècle de notre ère que le relais de poste de Lesmesnil (Meurthe-et-Moselle) s'installe dans la plaine de la Seille le long de la voie romaine qui relie Metz à Scarponne (Schembri et al., 2011). De nombreuses zones humides comme le bassin d'Outremont (Lucy, Moselle; Ollive et al., 2016), les sites de Haut-Clocher, Solgne, SaintEpvre, Peltre etc. (Gebhardt et al., 2014) sont investies à cette même époque. Toutefois, en raison de leur impréci- sion, les calages chronologiques par la céramique et les datations ${ }^{14} \mathrm{C}$ sur charbons de bois de structures archéologiques ne permettent pas d'appréhender les cycles pluriannuels ou saisonniers de sécheresse et d'humidité. Or, les fentes de sécheresse peuvent se former très rapidement à l'occasion d'un déficit saisonnier en eaux météoriques surtout dans les secteurs à forte pression agricole. Il est par ailleurs difficile de comparer des données météorologiques régionales car certains micro-terroirs peuvent souffrir de contextes pédo-climatiques négatifs, plus favorables que d'autres à la formation de fentes de dessiccation.

Cet optimum climatique n'a sans doute pas été l'unique déclencheur de l'occupation des zones humides. La gestion de l'excès d'eau a été très tôt un problème à résoudre pour développer l'agriculture et les sols lorrains ont sans doute été plus largement influencés par une longue activité agricole que par une évolution naturelle (Jacquin et Florentin, 1988; Florentin, 2005; Jamagne, 2011). Ainsi, le besoin de terres agricoles et l'amélioration des méthodes culturales ont pu accélérer la conquête des zones humides en cours d'assèchement naturel. Le drainage, bien connu à la période antique, a certainement pu accélérer la dégradation des luvisols. Dès 60 avant notre ère, le traité $D e$ l'agriculture (Columelle, 2002), ventait déjà les bienfaits du drainage pour dessécher les "terrains friables ", comme ce pourrait être le cas des sols limono-argileux. Il conseille le creusement de "fossés cachés " qui iraient se vider dans des fossés plus grands et ouverts. Le collecteur de drain de Bernolsheim qui servait aussi d'approvisionnement en eau pour le bétail (Fleischer et al., 2011; Leprovost et Fleischer, 2014; Fleischer et Leprovost, 2015), les drains en tuiles percées trouvés à Yutz (Blaising et al., 2009; Blaising 2013; Blaising et Gebhardt, 2014; Blaising, 2016), le drain de Haut-Clocher (Jude et al., 2012), confirment ces pratiques dès l'Antiquité. En Provence, l'implantation d'un réseau de drains, également attribué à cette dernière période, a permis d'assécher les dolines marécageuses du massif de la Sainte Victoire (Leveau, 2012). La dégradation des sols peut donc bien être liée à une baisse hydrique rapide à la faveur d'un aléa climatique pluri-décennal moins pluvieux, accéléré par du drainage agricole dès la période gallo-romaine.

\section{Perspectives}

À Bouxières-sous-Froidemont, l'étude micromorphologique a montré que ces fentes étaient postérieures au remaniement du BT (Gebhardt et al., 2014). Des études plus locales de dendrochroécologie (Tegel et Hakelberg, 2017) ou l'analyse spectrale de concrétions travertineuses historiques 
(Dubar, 2006) pourraient également aider à mieux discriminer cette rythmicité saisonnière de la pluviométrie dans le Grand Est. L'observation, l'inventaire, la cartographie ainsi que le calage chronologique de ces fentes blanchâtres en contexte de diagnostics et fouilles archéologiques préventives doivent devenir systématiques. Leur cartographie pourrait être incluse dans le cadre de la constitution d'une base de données sur les agrosystèmes anciens à l'échelle régionale ou micro-régionale (Camizulli et al., 2015). En parallèle, pour faire le lien avec des pratiques agraires anciennes liées à l'assèchement des sols, une attention particulière devra être portée aux modes de drainage (modernes, historiques ou protohistoriques). Enfin, pour mieux cibler d'éventuels épisodes de sécheresse antique et/ou de baisse du niveau de la nappe phréatique dans le Grand Est, il serait utile d'établir une chrono-cartographie des données historiques et botaniques, de la divagation des gués et des occupations en bord de rivière, des moulins, des voies, des ports, des pêcheries... Si l'hypothèse climatique se confirme, en affinant les observations pédo-stratigraphiques, nous pourrions nous orienter vers la caractérisation d'un niveau repère antique. Il serait alors d'une grande utilité dans la détermination d'un cadre chronologique sur le terrain en archéologie préventive dans le Grand Est. Enfin, mieux définir la variabilité et l'extension spatiale de sécheresse(s) au cours de l'Antiquité dans l'Est de la France serait un apport non négligeable des archives du sol aux rares données narratives connues de cette époque (Gebhardt, et al., 2017).

\section{ConCLUSION}

Le développement, au cours de l'Holocène dans certains sols du Grand Est, de réseaux polygonaux blanchâtres est donc attribué à un stress hydrique associé à l'assèchement de sédiments limono-argileux saturés en eau. L'étude détaillée de ces réseaux à partir de 4 sites archéologiques a permis leur calage chronologique au début de l'Antiquité. En apportant un cadre chronologique à la dégradation des luvisols, l'observation de ce réseau de fentes en contexte archéologique contribue également à la réflexion sur question de la vitesse de développement de la pédogenèse. À l'avenir, une observation plus approfondie de ces réseaux lors de diverses opérations archéologiques à l'échelle régionale, et sous réserve d'une confirmation de l'homogénéité de leur calage chronologique, pourrait permettre de proposer l'horizon d'apparition de ces réseaux comme un niveau repère du début de l'Antiquité pour les futurs diagnostics archéologiques. Enfin, une première confrontation de ces observations aux données climatiques, historiques et paléo-environnementales disponibles pour la région, permet de suggérer une origine climato-anthropique de cet épisode d'assèchement des sols bien marqué régionalement dans tout le Grand Est.

Cet article, issus de données acquises sur plus d'une dizaine d'années en contexte d'archéologie préventive (c'est à dire au gré de l'aménagement du territoire) est à considérer comme l'étape préliminaire d'une recherche qu'il reste à approfondir dans le cadre d'un programme scientifique plus ciblé.

\section{Bibliographie}

Andrieux E., Bertran P., Antoine P., Deschodt L., Lenoble L. A., Coutard S. et collaborateurs, 2016. Database of Pleistocene periglacial features in France: description of the online version. Quaternaire, 27, (4), p. 329-339.

Baize D., Girard M.-C., 1992. Le référentiel pédologique, principaux sols d'Europe, INRA, Techniques et Pratiques.

Baize D., 2008. Les grands processus pédogénétiques du territoire français. Zoom sur le processus d'éluviation verticale de particules argileuses menant à la formation des luvisols, INRA, Orléans. De [http://www.denis-baize.fr/documents/ZOOM-LUVISOLS. pdf], consulté en juin 2017.

Baize D., Girard M.-C., 2009. Le référentiel pédologique, AFES, Éditions Quae, coll. "Savoir Faire».

Baize, D., Jamagne M., 2009. Les processus de transfert de particules argileuses menant à la formation des luvisols et des planosols et contribuant à d'autres pédogenèses, INRA, Orléans. De [http:// www.denis-baize.fr/documents/Baize-JES-2009-ORAL.pdf], consulté en juin 2017.

Bégin P.-L., 2007. Fentes de retrait du sol et amas de fumier, rapport de la direction de l'Environnement et du développement durable. Bibliothèque et archives nationales du Québec. De [http://www.agrireseau.net/agroenvironnement/documents/ RdeL\%20FentesDeRetrait.pdf, consulté en juin 2017].

Bertran P., Andrieux E., Antoine P., Deschodt L., Font M., Sicilia D., 2017. Pleistocene involutions and patterned ground in France: Examples and analysis using GIS Database. Permafrost and Periglacial Processes, DOI:10.1002/ppp1957.

Blaising J.-M., Baccega S., Michel K., 2009. Yutz (Moselle), ZAC du Vieux Bourg. Rapport de fouilles, Metz, Inrap GEN.

Blaising J.-M., 2013. Yutz (Moselle), aéroparc : une occupation de l'Âge du Fer, de l'Antiquité et structures agraires modernes et contemporaines. Rapport de fouilles, Metz, INRAP.

Blaising J.-M., Gebhardt A., 2014. Yutz (Moselle), résidences de L'Ambanie : occupation de l'espace de l'Antiquité au XX' s. à proximité de l'établissement antique et du village disparu de HauteYutz. Rapport de fouilles, Metz, Inrap GEN. 
Blaising J.-M., 2016. Évacuer l'eau des champs, solutions antiques et contemporaines. Villages lorrains, $157: 39-30$.

Blanalt J.-G., Vogt H., Maire G., 1972. Carte géologique de la France au 1/50000', Brumath-Drusenheim [carte géologique], éch. 1/50000', Orléans, BRGM.

Blight G. E., 2009. Desiccation of soil by vegetation and potential interaction with buildings - a field study. Journal of the South African Institution of Civil Engineering, 51 (2), p. 1-17.

Bravard J.-P., 1997. Géo-archéologie des vallées alluviales du Rhône-Alpes depuis le Tardiglaciaire. In Bravard J.-P. et Prestreau M. (dir.). Dynamique du paysage, entretiens de géoarchéologie, Lyon, novembre 1995, DARA, p. 129-150.

Büntgen U., Tegel W., Nicolussi K., McCormick M., Trouet V., Kaplan J. O., Herzig F., Heussner K.-U., Wanner H., Luterbacher J., Esper J., 2011. 2500 years of European climate variability and Human Susceptibility. Science, 331, p. 578-582.

Caillier M., 1977. Étude chronoséquentielle des sols sur terrasses alluviales de la Moselle. Genèse et évolution des sols lessivés glossiques, thèse de doctorat, pédologie, université de Nancy I.

Camizuli E., Petit C., Bernigaud N., Reddé M., 2015. Principes méthodologiques pour caractériser des agrosystèmes antiques. Exploitation des bases de données actuelles. In Giligny F. et Tsirtsoni Z. (dir). Environnement et Sociétés. Les Nouvelles de l'archéologie, 142, p. 20-26.

Carcaud N, 1992. Remplissage des fonds de vallée de la Moselle et de la Meurthe en Lorraine sédimentaire, thèse de géographie, université de Nancy 2, France.

Columelle, 2002. De l'agriculture, Errance.

Cordier S., Harmand D., Losson B., Beiner M., 2004. Alluviation of the Meurthe and Moselle valleys (Eastern Paris Basin, France), lithological contribution to the study of the Moselle capture and Pleistocene climatic fluctuations. Quaternaire, 15 (1-2), p. 65-76.

Deffressigne S., Adam F., Forelle L., Gebhardt A., Pillard-Jude C., Vidal P., Zanatta S., 2012. Un micro-terroir de la fin de l'Âge du Bronze à la période antique. Vol. 2, annexes et inventaires. Rapport d'opérations, Metz, Inrap GEN.

Delhoume J.-P., Montana C., Cornet A., 1988. Vegetation patterns and soils in the Mapimi Bolson (Chihuahuan Desert - Mexico), II. Polygonal patterns. Contributed Papers of the Second Symposium on Resources of the Chibuahuan Desert Region: United States and Mexico (October 1983), The Chihuahuan Desert Research Institute. Alpine, Texas, USA, p. 1-14.

Dohr M., Gebhardt A. 2008. Remomeix (Vosges), les Mennes Hières, phase 2. Rapport de diagnostic, Metz, Inrap GEN.

Dohr M., Forelle L., Gebhardt A., Pillard-Jude C., 2014. Occupation du sol le long de la via Aggripa : Bouxières-sousFroidmont (Meurthe-et-Moselle), Tournailles de Narbois et Le Tremble : Lesménils (Meurthe-et-Moselle), Chêne Brûlé ZAC
Bouxières-sous-Froidmont-Lesménils. Rapport de diagnostic, Metz, Inrap GEN.

Dubar M., 2006. Approche climatique de la période romaine dans l'est du Var : recherche et analyse des composantes périodiques sur un concrétionnement centennal $\left(\mathrm{I}^{\mathrm{er}}-\mathrm{II}^{\mathrm{e}}\right.$ s. de notre ère). Archéosciences, 30, p. 163-171.

Duchaufour Ph., 1983. Pédogénèse et classification. Pédologie, t. 1, Paris, Masson.

Ewertowski M., 2009. Ice-wedge pseudomorphs and frost-cracking structures in Weichselian sediments, central-west Poland Permafrost. Periglacial Processes, 20, p. 316-330, DOI:10.1002/ ppp. 657.

Flageollet J.-C., 2002. Sur les traces des glaciers vosgiens. CNRS Édition.

Fleischer F., Leprovost C., Peter C., 2011. La fouille archéologique de la zone nord de la future plateforme départementale d'activité de Brumath entre Bernolsheim et Mommenheim. Bulletin de la Société d'histoire et d'archéologie de Brumath et des environs, 39, p. 25-28.

Fleischer F., Leprovost C., 2015. 110 Hektar Siedlungsgeschichte. Archäologische Untersuchungen im Umfeld des antiken Brocomagus-Brumath (Bas-Rhin, Elsass). Beiträge des internationalen Symposiums zur Archäologie in der Großregion in der Europäischen Akademie Otzenhausen vom 7-9 März, p. 47-66.

Florentin L., 2005. Notice de la carte pédologique de France au 1/1000000, feuille de Metz, INRA.

Forelle L., Gebhardt A., 2012. Moncel-lès-Lunéville (Meurthe-etMoselle), Betaigne. Rapport de diagnostic, Metz, Inrap GEN.

Gebhardt A., 2004. Compte rendu géomorphologique. In Antoine X. (dir.). Illange-Bertrange (Moselle), Mégazone. Rapport de diagnostic, INRAP-GEN, DRAC Lorraine.

Gebhardt A., Occhietti S., Fechner K., 2014. Grandes phases de pédogenèse, d'érosion et d'anthropisation des sols au cours de la seconde moitié de l'Holocène en Lorraine (France). Archéosciences, 8, p. 7-29.

Gebhardt A., Robin V., Blaising J.-M., Boulanger K., Nüsslein A., 2017. Variabilité et extension spatiale de sécheresse(s) au cours du début de l'Antiquité dans le Grand Est de la France. Apport des archives du sol aux rares données narratives connues. In Metzger A. et De Jong C. (dir.). Adaptation et résilience aux sécheresses : perspectives historiques en Europe et alentours, Strasbourg, $1^{\text {er }}-2$ juin 2017, 24.

Goehring L, Conroy R., Akhter A., Clegg W. J., Routh A. F., 2010. Evolution of mudcrack patterns during repeated drying cycles. Soft Matter, 6 (15), p. 3562-3567, DOI:org/10.1039/ B922206E.

Gücker D., Gebhardt A., 2007. Remomeix (Vosges), les Mennes Hières, phase 2 (voirie). Rapport de diagnostic, Metz, Inrap GEN. 
Gury M., 1990. Genèse et fonctionnement actuel des pseudogleys podzoliques sur terrasses alluviales dans l'Est de la France, thèse d'état de l'université de Nancy I.

Holzhauser H., Magny M., Zumbuhl H., 2005. Glacier and lake level variations in west-central Europe over the last 3500 years. The Holocene, 15 (6), p. 789-801.

IUSS Working Group WRB, 2015. World Reference Base for Soil Resources 2014, update 2015. International soil classification system for naming soils and creating legends for soil maps. World Soil Resources Reports 106, FAO, Rome.

Jacquin F., Florentin L., 1988. Atlas des sols de Lorraine, ENSAIA, PU Nancy.

Jamagne M. 1973. Contribution à l'étude pédogénétique des formations loessiques du Nord de la France, thèse, faculté de Sciences agronomiques, Gembloux, Belgique.

Jamagne M., 2011. Grands paysages pédologiques de France, coll. Synthèses INRA, Éditions Quae, Versailles.

Jeandemange S., 2006. Cerville (Meurthe-et-Moselle), VC $n^{\circ} 5$ de Cerville à Laneuvelotte. Rapport de diagnostic, Metz, Inrap GEN.

Jeandemange S., Ferraresso Y., Meyer N., Prouteau R., 2013. Phalsbourg (Moselle), ZAC Louvois - secteur péage, phase 1. Rapport intermédiaire. Rapport de diagnostic, Metz, Inrap GEN. Jude R., Gebhardt A., 2006. Remomeix (Vosges), les Mennes Hières. Rapport de diagnostic, Metz, Inrap GEN.

Jude R., Gebhardt A., Wietold J., 2012. Étude géomorphologique. In Jude R. (dir.). Haut-Clocher (Moselle), Ersterbach, LGV Est, site 22, anthropisation et gestion d'un vallon, de l'Antiquité au premier Moyen Âge. Rapport de fouille archéologique, Metz, Inrap GEN.

Kishné A. S., Morgan C. L. S., Ge Y., Miller W. L., 2010. Antecedent soil moisture affecting surface cracking of a vertisol in field conditons. Geoderma, 157, p. 109-117.

Langohr R., 2010. Quelques facteurs édaphiques dans l'écosystème forêt de Soignes. Forêts Wallonnes, 105, p. 3-14.

Lenoble A., Bertran P., Mercier N., Sitza L., 2012. Le site du lac Bleu et la question de l'extension du pergélisol en France au Pléistocène supérieur. In Bertran P. et Lenoble A. (dir.). Quaternaire continental d'Aquitaine : données récentes, livret guide excursion de l'AFEQ, p. 109-121.

Leprovost C., Fleischer F., 2014. La plateforme départementale d'activités de la région de Brumath et environs. Résultats des fouilles de 2014. Bulletin de la Société d'histoire et d'archéologie de Brumath et des environs, 42, p. 25-28.

Leveau Ph., 2012. Le drainage de dépressions fermées en Provence et dans la vallée du Rhône. Un bilan et les enjeux de la recherche. In Ropiot V., Puig C. et Mazière F. (dir.). Les plaines littorales en Méditerranée nord-occidentale. Regards croisés d'histoire, d'archéologie et de géographie, de la Protohistoire au Moyen Âge, p. 169-194.
Leroux J., 2007. Introduction à la géologie de l'Alsace-Lorraine et des régions limitrophes. In Cordier S., Harmand D. et Occhietti S. (dir.). Livret guide de l'excursion AFEQ des 7-9 juin 2007, Lorraine, Luxembourg, Rhénanie-Palatinat, Sarre, p. 27-34.

Lexa-Chomard A., Pautrot C., 2006. Géologie et géographie de la Lorraine, Éditions Serpenoises.

Lozet J., Mathieu C., 1997. Dictionnaire des sciences du sol, Lavoisier., coll. « Tec et Doc ».

McCormick M., Büntgen U., Cane M. A., Cook E. R., Harper K., Huybers P., Litt Th., Manning S. W., Mayewski P. A., More A. F. M., Nicolussi K., Tegel W., 2012. Climate change during and after the roman Empire: Reconstructing the past from Scientific and historical Evidence. Journal of Interdisciplinary History, 43 (2), p. 1969-220.

Meyer N., Blaser F., Delaunay L., Forelle L., Gebhardt A., 2015. Sionne, Les Plates Terres: Coussey et Sionne (Vosges), Launot et Longues Royes : projet de gravière Société Paul Calin phase 3. Rapport de diagnostic, Metz, Inrap GEN.

Mondy M., Billaudeau E., Gebhardt A., Frauciel M., 2015. Hayange (Moselle), lotissement Les Résidences de Marspich, rue de Leyrange : de l'occupation de La Tène finale au hameau du haut Moyen Âge et Moyen Âge classique. Rapport de fouilles, Metz, Inrap GEN.

Montagne D., 2006. Impact de la mise en culture et du drainage sur l'évolution récente des sols : cas des luvisols dégradés de l'Yonne, thèse de doctorat en sciences du sol, INRA, Orléans, France.

Ollive V., Ernst Th., Franck J., Meyer N., Thomashausen L., Viller S., 2016. L'érosion agricole d'un petit bassin versant lorrain depuis 2000 ans : géoarchéologie de la LGV est-européenne. Quaternaire, 27 (1), p. 27-37.

Peron H., Laloui L., Hueckels T., 2009. Desiccation cracking of soils. EJECE, Failure in multiphase geomaterial, DOI: 10.3166/ EJECE.13.869-888.

Peron H., Laloui L., Hu L.-B., 2012. Formation of drying crack patterns in soils: a deterministic approach. Acta Geotechnica, DOI: $10.1007 / \mathrm{s} 11440-012-0184-5$.

Petitdidier M.-P., 2018. Terville, ZAC du PAT. Rapport de fouilles, Metz, Inrap GEN.

Plaisance G., 1954. Observation sur les sous-sols marmoroïdes de certains sols de limons. Revue géographique de Lyon, 29 (2), p. 165-166.

Rachet V., Gebhardt A., 2010. La Salle (Vosges), le Champs des Tocs. Rapport de diagnostic, Metz, Inrap.

Rachet V., Gebhardt A., 2012. Ancerville (Meuse), clos Le Prêtre. Rapport de diagnostic, Metz, Inrap GEN.

Rachet V., Gebhardt A., Delaunay L., 2017. Chamagne, le bois de Genet. Rapport de diagnostic, Metz INRAP.

Reeve M. J., Hall D. G. M., Bullock P., 1980. The Effect of Soil Composition and Environmental Factors on the Shrinkage of 
Some Clayey British soils. European Journal of Soil Science, 31 (3), p. 429-442.

Risacher F., Fritz B., 1992. Mise en évidence d'une phase climatique holocène extrêmement aride dans l'Altiplano central, par la présence de la polyhalite dans le salar de Uyuni (Bolivie). C. r. de l'Académie des sciences, Paris, 314 (II), p. 1371-1377.

Sauer D., Schülli-Mauer I., Sperstad R., Sorensen R., 2013. Micromorphological characteristics reflecting soil-forming processes during Albeluvisol development in S. Norway. Spanish Journal of Soil Science, 3 (2), DOI:10:3232/JSS.2013. V3.N2.03.

Schembri F., Cabart H., Feller M., Gebhardt A., 2011. Lesmenils (Meurthe-et-Moselle), le Chêne Brûlé, fouilles TGV Est site 188, lot 34. Rapport de fouilles, Metz, Inrap GEN.

Schwab H., 2003. Ponts et ports romains sur la Broye inférieure et la Thielle moyenne. Archéologie de la $2^{e}$ correction des eaux du Jura, vol. 4 Fribourg, Éditions universitaires, coll. "Archéologie fribourgeoise ", p. 17.

Sell Y., Berchtold J.-P., Callot H., Hoff M., Gall J.-C., Walter J.-M., 1998. L'Alsace et les Vosges, Paris, Delachaux et Niestlé, coll. "La Bibliothèque du naturaliste ».

Tegel W., Hakelberg D., 2017. Jahringdaten archäologischer Holzfunde als Klima- und Umweltarchiv. Geogeraphische Rundschau, 7-8, p. 8-15.

Van Geel B., Magny M., 2002. Mise en évidence d'un forçage solaire du climat à partir de données paléo-écologiques et archéologiques : la transition Subboréal-Subatlantique. In
Richard H., Vignot A. (dir.). Équilibres et ruptures dans les écosystèmes depuis 20000 ans en Europe de l'Ouest. Actes du colloque de Besançon, septembre 2000.

Van Vliet-Lanoë B., Langohr R., 1981. Correlation between fragipans and permafrost with special reference to Weichel silty deposits in Belgium and Northern France. Catena, 8, p. $137-$ 154.

Van Vliet-Lanoë B., Coutard J.-P., Pissart A., 1984. Structures caused by repeated freezing and various loamy sediments: a comparison of active, fossil and experimental data. Earth Surface Processes and Landforms, 9, p. 553-565.

Van Vliet-Lanoë B., 1988. Le rôle de la glace de ségrégation dans les formations superficielles de l'Europe de l'Ouest. Processus et héritages, thèse d'état, université de Paris I-Sorbonne, Caen, Editec.

Van Vliet-Lanoë B., 2006. Deformation in the active layer related with ice/soil wedge growth and decay in present day artic. Paleoclimatic implication. Annales de la Société géologique du Nord, 12 (1), p. 81-95.

Vogt. H. 1992. Le relief en Alsace. Étude géomorphologique du rebord sud-occidental du fossé rhénan, Oberlin, Strasbourg.

Wahl L., 2007. Le climat de la Lorraine. In Cordier S., Harmand D., Occhietti S. (dir.). Livret guide de l'excursion AFEQ des 7-9 juin 2007, Lorraine, Luxembourg, Rhénanie-Palatinat, Sarre, p. 85-89.

Weinberger R., 1999. Initiation and growth of cracks during desiccation of stratified muddy sediments. Journal of structural geo$\log y, 21$, p. 379-386. 